\title{
DIRECTIONAL GROWTH OF L-GLUTAMIC ACID HYDROCHLORIDE (LGHCL) SINGLE CRYSTALS GROWN BY CONVENTIONAL AND SANKARANARAYANAN-RAMASAMY (SR) METHOD: A COMPARATIVE STUDY BY MEANS OF OPTICAL, LDT, PHOTOCONDUCTIVITY, PHOTO ACOUSTIC AND POLARIZABILITY PROPERTIES
}

\author{
Jayanthi L, Prabavathi N*
}

Department of Physics, Sri Sarada College for Women (Autonomous), Salem-636 016, Tamilnadu, India

\section{A R T I C L E I N F O}

\section{Article History:}

Received $4^{\text {th }}$ February, 2017

Received in revised form $10^{\text {th }}$ March, 2017

Accepted $19^{\text {th }}$ April, 2017

Published online $28^{\text {th }}$ May, 2017

\section{Key words:}

Directional Growth (001) Orientation; LDT; Photoconductivity; Electronic Polarizability

\begin{abstract}
A B S T R A C T
Growth of non-linear optical single crystals of Lglutamic acid hydrochloride by Sankaranarayana - Ramasamy (SR) method. The (001) face was chosen for unidirectional growth. The grown crystals was confirmed by single crystal X-ray diffraction (SXRD) and characterized using optical, Laser damage threshold, Photoconductivity, Photoacoustic, electronic polarizability and their properties were compared by conventional method. Results show that the SR method grown crystal exhibit higher optical, laser damage threshold, photoconductivity due to relatively high- crystalline perfection. The electronic polarizability and photoacoustic were also discussed for the title compound.
\end{abstract}

Copyright $(\mathbf{C} 2017$ Jayanthi L and Prabavathi N. This is an open access article distributed under the Creative Commons Attribution License, which permits unrestricted use, distribution, and reproduction in any medium, provided the original work is properly cited.

\section{INTRODUCTION}

In the recent past decade, semi organic nonlinear (NLO) optical single crystals have emerged as one of the most attractive fields of research in view of potential applications in the area of optoelectronics, photonics, telecommunication, optical computing, optical storage and optical information process[1,2]. For practical NLO applications, the crystals must possess good optical transparency, high laser damage threshold, high mechanical strength and so on. These restrictions are satisfied by the semi organic single crystals [3]. The conventional method is mostly used for the convenience, simplicity and the possible avoidance of complex growth apparatus. There are different techniques are available to grow bulk size single crystals. The Sankaranarayanan-Ramasamy (SR) method is the best way to grow bulk size single crystal in solution technique for device applications [4]. The main advantages of SR method are simple experimental setup, unidirectional (single plane) growth, high solution-crystal conversion, minimum thermal stresses on the crystal during growth and avoidance of the microbial growth [5]. Finally, we can achieve bulk size single crystals with required specific orientation. The literature information concerning with growth and characterization of L-Glutamic acid hydrochloride (LGHCL) are reviewed here:

*Corresponding author: Prabavathi N

Department of Physics, Sri Sarada College for Women (Autonomous), Salem-636 016, Tamilnadu, India
Bairava Ganesh et al, [6], Selvaraju et al [7], Sathyalakshmi et al [8] and Uma et al [9] reported the title compounds for UV-transmittance, mechanical, dielectric and SHG measurement. To the best of our knowledge, there are no earlier reports have encountered in the literature survey on systematic comparative investigations on photoluminescent, Laser damage threshold, Photoconductivity, Photoacoustic and electronic polarizability by conventional and SR method for LGHCL crystal.

\section{Experimental procedure and crystal growth}

L-glutamic acid hydrochloride (LGHCL) was synthesised from the raw materials of L-glutamic acid and hydrochloric acid (Fig.1) taken in stoichiometric ratio of $1: 1$ and water is used as a solvent [10].

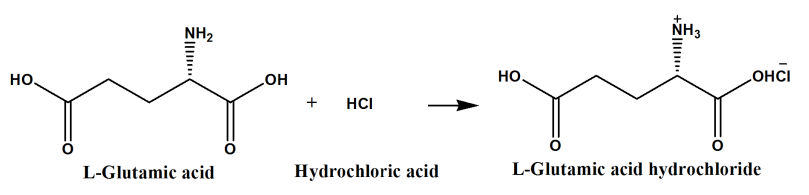

Figure 1 Reaction scheme of LGHCL

The synthesized materials have been purified by repeated recrystallization process to eliminate any impurities in the grown crystals using same solvent. The optically good transparent LGHCL crystals of size $12 \times 5 \times 3 \mathrm{~mm}^{3}$ were grown from conventional and it is shown in Fig.2(a). The morphology of LGHCL has been indexed by using WinXmorph software (Fig.2 (b)). Based on the morphology 
of the conventionally grown LGHCL crystal, the (001) face was selected. According to the Periodic bond chain (PBC) theory, the growth rate of crystal at particular orientation is directly proportional to attachment energy. The faces which have higher attachment energy grow faster than the other orientation (Wang Yan et al 2005) [11]. The fast growth orientation (001) was used for growth of large size LGHCL crystals.

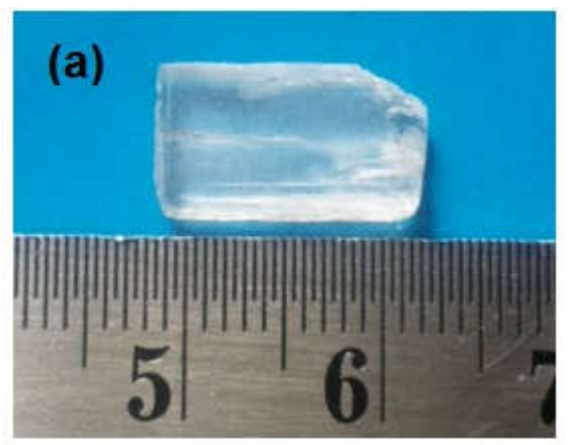

Figure a

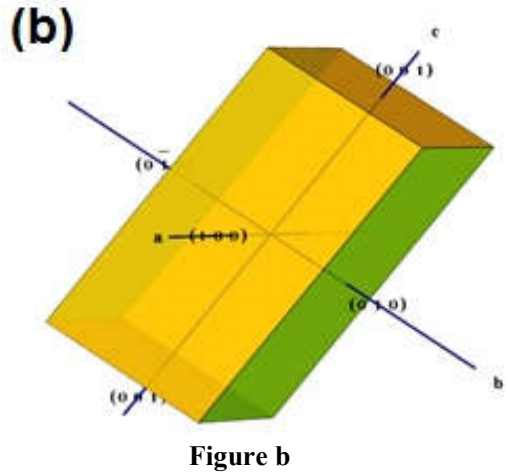

Figure 2 (a) conventionally grown crystal and (b) Its morphology

The seed crystal was mounted at the bottom of the glass ampoule along (001) orientation. The saturated homogenous solution was prepared and transferred into seed mounted glass ampoule without any disturbance to seed and positioned in an assembled water bath. The suitable temperature was provided by the ring heater to avoid the temperature fluctuation around the ampoule. The perfect growth has been achieved by optimization of temperature. The temperature at the top of the ampoule was $40^{\circ} \mathrm{C}$ for solvent evaporation and at the bottom is $34^{\circ} \mathrm{C}$ for growing crystal. After 10 days, the mounted seed crystal started to grow at the bottom of the ampoule. Under this condition the growth system was kept undisturbed for a long period. Crystals of $130 \mathrm{~mm}$ in length and $20 \mathrm{~mm}$ in diameter have been grown successfully within a period of 60 days. The Fig.3 (a) and (b) shows the SR method grownLGHCL single crystal with the ampoule as well as cut and polished crystal wafers respectively.

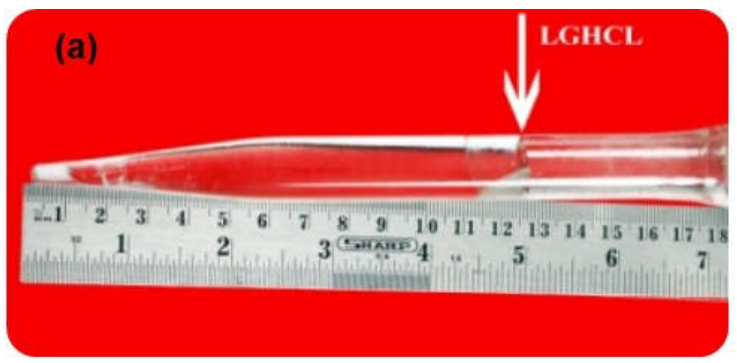

Figure 3 a

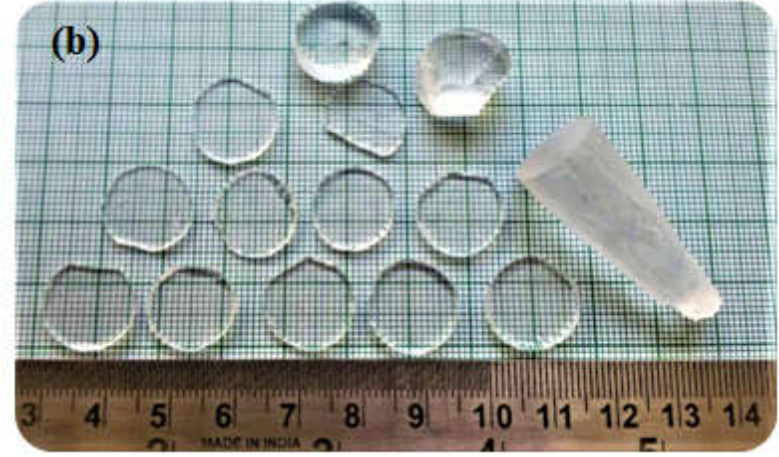

Figure 3 b

Figure 3 (a) SR method grown crystal with the glass ampoule and (b) Cut and polished LGHCL crystal

\section{Characterization technique}

The grown LGHCL single crystal was subjected to various characterization studies. The Bruker AXS Kappa APEX II CCD Diffractometer, equipped with monochromatic Mo $\mathrm{K}_{\alpha}$ radiation $(\lambda=0.710 \AA)$ was used to estimate the cell parameters. UV-Vis NIR transmission spectrum studies were carried out in the range 190-1200 nm using Perkin-Elmer Lambda-35 spectrophotometer. The photoluminescence spectrum was recorded with Shimadzu Spectrofluorophotometer R.F-5031 PC series with Xenon arc lamp as the excitation source. The conductivity behaviour was carried out on a cut and polished LGHCL sample using KEITHLEY 6487 picoammeter in the presence of DC electric field at room temperature. The photoacoustic and electronic polarizability were also determined. Laser damage threshold studies were carried out using Q-switched Nd: YAG laser operating at $1064 \mathrm{~nm}$ radiation.

\section{RESULTS AND DISCUSSIONS}

\section{X-ray Diffraction}

The single crystal XRD data confirms that, the LGHCL crystal belongs to orthorhombic crystal system having noncentrosymmetric nature with space group $\mathrm{P} 2{ }_{1} 2{ }_{1} 2_{1}$ and the cell parameters are $a=13.23(8) \AA, b=11.68(1) \AA$ and $c=5.161(5)$ $\AA$ with Volume $\mathrm{V}=797.99 \AA^{3}$. The observed values are in good agreement with the reported values [10].

\section{Optical analysis}

\section{UV-Vis NIR analysis}

The optical transmission spectrum gives valuable information about the band gap energy, band structure and the

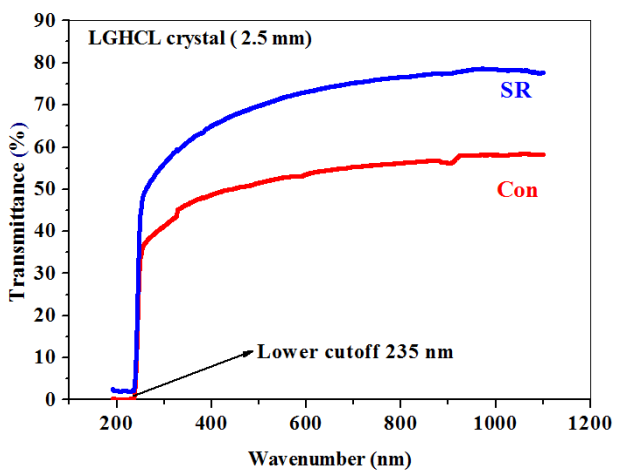

Figure 4 UV-Vis NIR spectrum of conventional and SR method grown crystals 
transparency window which are very essential for the device in optoelectronic applications [12]. The transmittance spectrum was recorded in the wavelength range from 200$1100 \mathrm{~nm}$ and shown in Fig.4. The good and defect free sample with well-polished surface of conventional and SR grown LGHCL crystal along (001) orientation with identical thickness of $2.5 \mathrm{~mm}$ were employed. The LGHCL crystal has good transparency in the entire visible region. The absence of absorption of light in the visible region is an intrinsic property of all the amino acids. Absence of absorption in the entire region is theis the most desirable property of the materials possessing NLO activity. The cut off wavelength of both the method grown LGHCL crystal was found to be $235 \mathrm{~nm}$, which is sufficient for SHG. It was observed that the transmittance percentage of SR method grown crystal was 23 $\%$ higher than that of conventionally grown crystal. This may be attributed to reduced scattering centers from point and line defects. This illustrates the optical quality of the SR method grown crystal. The optical absorption co-efficient $(\alpha)$ was calculated using the relation,

$\alpha=\frac{2.3026}{t} \log _{10}\left(\frac{100}{T}\right)$

where ' $\mathrm{T}$ ' is the transmittance (\%) and ' $\mathrm{t}$ ' is the thickness of the crystal $(2.5 \mathrm{~mm})$. In the high photon energy region, the energy dependence of the absorption co-efficient suggests the occurrence of a direct band gap of the crystal obeying the following equation

$$
(\alpha h v)=A\left(h v-E_{g}\right)^{\frac{1}{2}}
$$

where ' $A$ ' is a constant, ' $E_{g}$ ' the optical band gap and ' $h v$ ' is the incident photon energy. The Tauc's graph [13] plotted between variation of $(\alpha h v)^{2}$ versus (hv) is shown in Fig. 5. Using this graph the band gap energy $\mathrm{E}_{\mathrm{g}}$ was calculated by extrapolating the linear portion near the onset of absorption edge to the energy axis and is equal to $5.05 \mathrm{eV}$, which is consistent with theoretically calculated value $5.2 \mathrm{eV}$ using the relation $\mathrm{Eg}=\mathrm{h} \nu / \lambda_{\max }$.

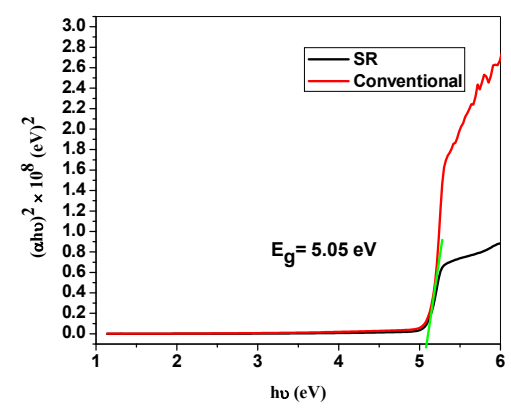

Figure 5 Band gap of LGHCL crystals

\section{Photoluminescence (PL) measurement}

The photoluminescence is highly depends on the crystallinity and structural perfection of the crystal. It is the spontaneous emission of light radiation by the absorption of photons. Although the excitation energy is typically in the form of photons, it could also be generated by an electric field or by ionizing radiation. The LGHCL crystal was excited at $300 \mathrm{~nm}$ and the emission spectrum was recorded in the range between
250-650 nm and shown in Fig.6. Generally, photoluminescence phenomenon is expected in aromatic molecules which contain multiple conjugated bonds leading to a high degree of resonance stability [14]. The sharp emission peak in the blue region observed at $480 \mathrm{~nm}$ for both conventional and SR method grown LGHCL crystal.

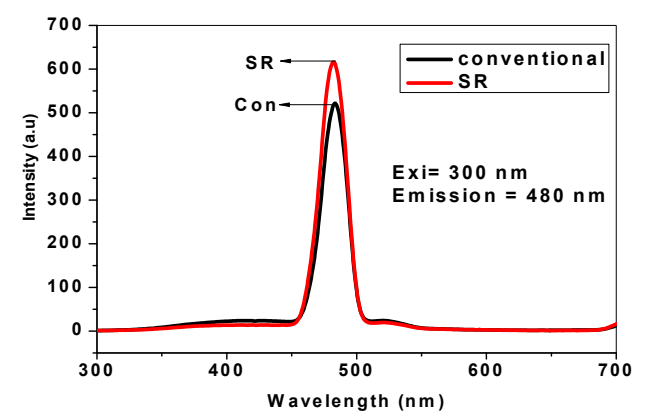

Figure 6 Photoluminescence of conventional and SR method grown crystal

The high peak intensity of the SR method grown crystal indicates its crystallinity and optical quality enrichment [15].

\section{Laser damage threshold (LDT)}

Laser damage threshold (LDT) analysis is very important because the functions of NLO devices such as second harmonic generation, electro-optic modulation, $\mathrm{THz}$ wave generation etc., mainly involve in the ability to sustain high intensity laser light. In the present study, a Q-switched $\mathrm{Nd}$ : YAG laser operating at wavelength of $1064 \mathrm{~nm}$ with $10 \mathrm{~Hz}$ repetition rate and pulse width of laser $10 \mathrm{~ns}$ was used. The multiple shots laser damage threshold measurements were made conventional and SR method grown crystals having 2.5 $\mathrm{mm}$ thickness. Initially $30 \mathrm{~mJ}$ was applied on the (001) surface of both the crystals and then gradually increased. For SR grown crystal when the laser energy was increased up to $427 \mathrm{~mJ}$, a visible damage and cracks have been observed. The diameter of the damage spotwas $893.04 \mu \mathrm{m}$, whereas for conventionally grown crystals the damage spot of diameter $1897.74 \mu \mathrm{m}$ was observed at $388 \mathrm{~mJ}$. The surface damage profile as observed through the optical microscope is shown in Fig.7 $(\mathrm{a}, \mathrm{b})$. The energy density $\left(\mathrm{P}_{\mathrm{d}}\right)$ was calculated by taking the ratio of input energy and area of the crystal exposed to laser radiation which is expressed in $\mathrm{GW} / \mathrm{cm}^{2}$ i.e.,
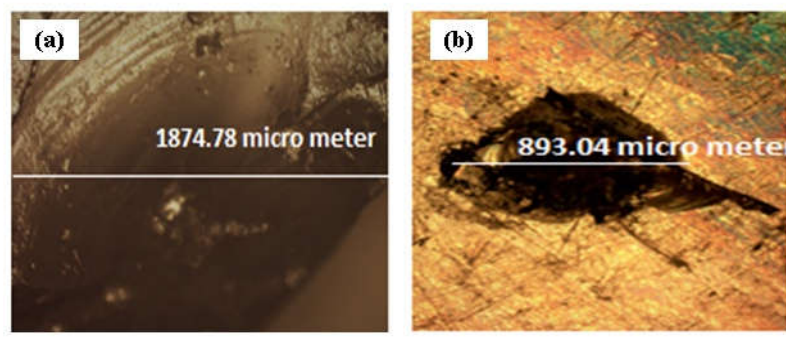

Figure 7 Laser damaged surface of (a) conventional and (b) SR method grown crystal

Power density $\left(\mathrm{P}_{\mathrm{d}}\right)=\frac{E}{\tau \pi r^{2}}$

(3)

where ' $E$ ' is the input energy density $(\mathrm{mJ})$, ' $\tau$ ' is the pulse width (ns) and ' $r$ ' is the radius of the damage spot $(\mu \mathrm{m})$ on the sample. The damage threshold were 
found to be $1.4 \mathrm{GW} / \mathrm{cm}^{2}$ and $6.8 \mathrm{GW} / \mathrm{cm}^{2}$ for the crystals grown by conventional and SR method respectively. High value of LDT indicates that SR method grown LGHCL crystal contains very low defects and is in tune with less EDP of $3.1 \times 10^{2} \mathrm{~cm}^{-2}$ shown in Fig. 8 (b). The low LDT value of conventionally grown LGHCL crystal suggests the high dislocation content in the crystal due to the presence of growth sector boundaries $\left(E P D=5.2 \times 10^{2} \mathrm{~cm}^{-2}\right.$ (Fig.8(a)). In $\mathrm{SR}$ method grown crystal there is no growth fluctuation/growth sector boundaries due to uniform growth rate. The calculated laser damage threshold value of LGHCL is higher than that of KDP, urea[16] and other standard NLO materials like $\mathrm{KNbO}_{3}, \mathrm{LiNbO}_{3}[17]$.
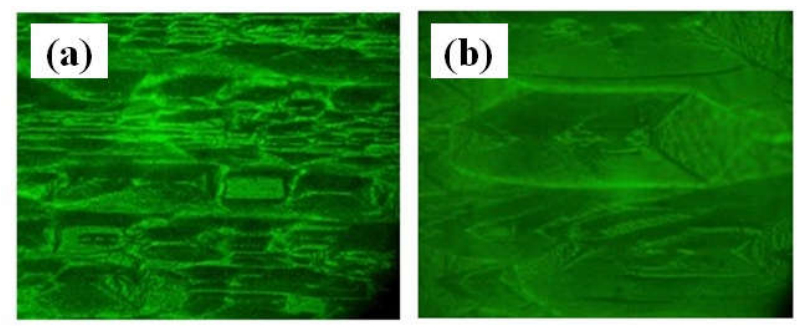

Figure 8 (a) Etch pit pattern of conventional and (b) Etch pit pattern of SR method grown crystal

\section{Photoconductivity}

When a material absorbs radiation of sufficient quantum energy there may be an increase or decrease of electrical conductivity called photoconductivity. The photoconductivity measurements of the grown LGHCL crystals were carried out at room temperature. Electrical contacts were made on the sample using silver paint and it was connected to the electrodes. The DC voltage applied to the electrodes and it was varying from 1-50 V with step voltage of $1 \mathrm{~V} / \mathrm{s}$ for the entire work. The light from halogen lamp of $50 \mathrm{~W}$ was focused on the sample.

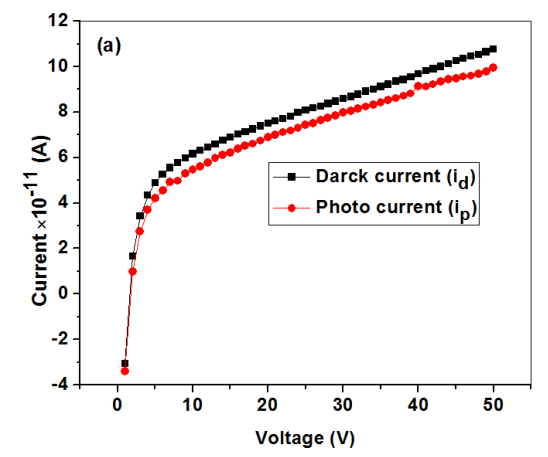

Figure 9 a

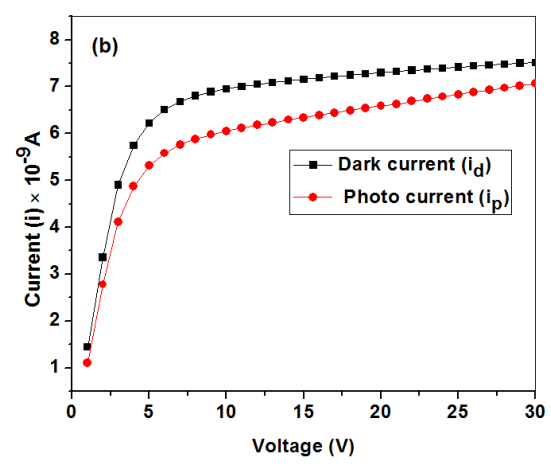

Figure 9 b

Figure 9 Photoconductivity of (a) conventionally grown crystal and (b) SR method grown crystal
Initially the dark current $\left(i_{d}\right)$ was recorded by keeping the sample unexposed to any radiation with respect to the applied voltage. As well as photocurrent $\left(i_{p}\right)$ was measured by applying the same voltage under illumination. Fig.9 (a) and (b) shows analysis for SR and conventional method which depicts the dark current is always higher than the photocurrent, thus confirming the negative photoconductivity nature. This may be due to decrease in either the number of charge carriers or their life time, when subjected to radiation. Decrease in life time with illumination could be due to the trapping process and increase in carrier velocity according to the relation:

$$
\tau=\left(\frac{1}{v s N}\right)
$$

where ' $v$ ' is the thermal velocity of the carriers, ' $\mathrm{s}$ ' is the capture cross section of the recombination centers and ' $\mathrm{N}$ ' is the carrier concentration. The phenomenon of negative photoconductivity was explained by Stockmann model [18]. The intense light continuously falls on the sample, the lifetime of the charge carriers could be decreases. As a result, the recombination of electrons and holes take place resulting in decrease in the number of mobile charge carriers, giving rise to negative photoconductivity. Negative photoconductivity materials can be used for UV and IR detector applications [19]. The SR method grown crystal has higher photosentivity than the conventionally grown crystal, as its depend upon the value of Ip, which is greater for SR method grown crystal.

\section{Photoacoustic analysis}

Photoacoustic spectroscopy (PAS) is the oldest form of photo thermal spectroscopy. It is a technique or mechanism of detecting and measuring the absorption coefficient of weakly absorbing or opaque and diffuse materials. In PAS, the irradiation of the sample contained in a closed gas cell produces temperature and pressure fluctuation in the gas which couples the light to the samples synchronous with the modulated source signal. A microphone placed in the gas cell picks up these fluctuations and an associated amplifying system produces an electrical signal whose amplitude and phase monitored over a range of wavelengths contain information about a wide range of optical phenomenon including optical absorption in highly transparent materials, non-linear absorption and localized defect characteristics [2022]. The unique advantage of PAS over other conventional spectroscopy is that it does not require optical detection of either transmitted or reflected.

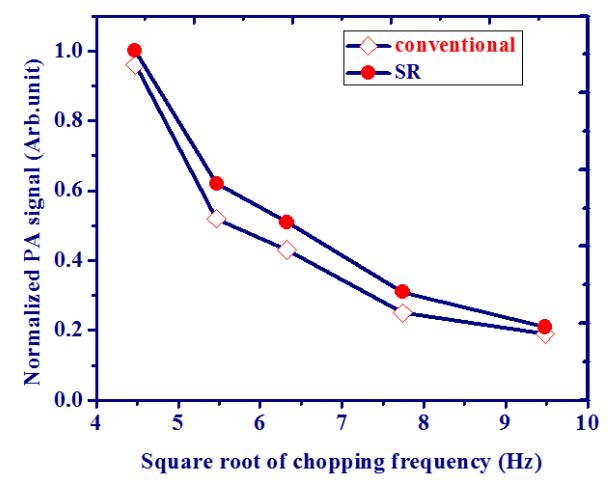

Figure 10 Photoacoustic study for conventional and SR method grown crystal 
The variation of photo acoustic signals with respect to chopping frequency from 20 to $90 \mathrm{~Hz}$ was measured for both the crystals. The normalised spectra of PA signal versus square root of chopping frequency are shown in Fig. 10. The thermal diffusivity is an important parameter for NLO material that describes the heat transport from the hot side to cold side. From PA analysis, the thermal diffusivity was derived by curve fitting method adopted by Barros and Mela [23]. The Fig.10 it illustrates, the conventionally grown crystal has lower thermal diffusivity value due to its crystalline defects.

\section{Electronic polarizability ( $\alpha$ )}

The parameter of electronic polarizability $(\alpha)$ of the material is essential for desired efficiency of nonlinear effect. Theoretically the valence electron Plasma energy, Penn gap, Fermi energy and electronic polarizability are depends upon the dielectric constant. The valence electron plasma energy $\left(\hbar \omega_{\mathrm{P}}\right)$ is given by [24]

$\hbar \omega_{p}=28.8\left(\frac{Z^{\prime} \times \rho}{M}\right)^{\frac{1}{2}}$

where the total number of valence electrons of LGHCL crystal is $Z^{\prime}=\left[\left(5 \times Z_{C}^{\prime}\right)+\left(10 \times Z_{H}^{\prime}\right)+\left(1 \times Z_{C l}^{\prime}\right)+\right.$ $\left.\left(1 \times Z_{N}^{\prime}\right)+\left(4 \times Z_{O}^{\prime}\right)\right]=66 .{ }^{\prime} M^{\prime}$ the molecular weight and ' $\rho$ ' is the density of LGHCL.

According to the Penn model [25], the average Penn gap ( $\left.E_{P}\right)$ and Fermi energy $\left(\mathrm{E}_{\mathrm{F}}\right)$ for LGHCL are calculated using the following relations:

$$
\begin{aligned}
& E_{P}=\frac{\hbar \omega_{p}}{\left(\varepsilon^{\prime}-1\right)^{\frac{1}{2}}} \\
& E_{F}=0.2948\left(\hbar \omega_{p}\right)^{\frac{1}{2}}
\end{aligned}
$$

where $\varepsilon^{\prime}$ is the dielectric constant. The dielectric constant for the SEST and SR method grown crystals are 50 and 55 respectively. The electronic polarizability $(\alpha)$ of the LGHCL crystal are calculated using the following relation [26]

$\alpha=\left[\frac{\left(\hbar \omega_{p}\right)^{2} S_{0}}{\left(\hbar \omega_{p}\right)^{2} S_{0}+3 E_{p}{ }^{2}}\right] \times \frac{M}{\rho} \times 0.396 \times 10^{-24} \mathrm{~cm}^{3}$

where, ' $\mathrm{S}_{0}$ ' is a constant for a particular material which is given by

$$
S_{0}=1-\left[\frac{E_{p}}{4 E_{F}}\right]+\frac{1}{3}\left[\frac{E_{p}}{4 E_{F}}\right]^{2}
$$

The value of electronic polarizability $(\alpha)$ is also confirmed by using the Clausius-Mossotti relation:

$$
\alpha=\frac{3 M}{4 \pi N_{A} \rho}\left(\frac{\varepsilon^{\prime}-1}{\varepsilon^{\prime}+2}\right)
$$

For SR grown crystal, the electronic polarizability is two times greater than that of KDP [27]. The calculated parameters are shown in Table 1.
Table 1 Electronic polarazibilty $(\alpha)$ results based on dielectric analysis

\begin{tabular}{cccc}
\hline Parameters & Conventiona] & SR & KDP [27] \\
\hline Plasma energy $\hbar \omega_{p}(\mathrm{eV})$ & 21.179 & 21.179 & 17.33 \\
Penn gap energy $\mathrm{E}_{\mathrm{P}}(\mathrm{eV})$ & 3.025 & 2.462 & 2.39 \\
Fermi energy $\mathrm{E}_{\mathrm{F}}(\mathrm{eV})$ & 17.274 & 17.274 & 12.02 \\
Electronic polarizability $(\alpha)$ & $4.542 \times 10^{-23}$ & $4.637 \times 10^{-23}$ & $2.14 \times 10^{-23}$ \\
using Penn analysis $\left(\mathrm{cm}^{3}\right)$ & & & \\
Electronic polarizability $(\alpha)$ & & & \\
using Clausius-Mossotti & $4.560 \times 10^{-23}$ & $4.650 \times 10^{-23}$ & $2.18 \times 10^{-23}$ \\
$\quad$ equation $\left(\mathrm{cm}^{3}\right)$ & & & \\
\hline
\end{tabular}

\section{CONCLUSIONS}

The optically transparent semi-organic NLO single crystal of L-Glutamic acid hydrochloride (LGHCL) has been successfully grown by conventional method using water as solvent. High quality (001) directed LGHCL crystal of dimension $130 \mathrm{~mm}$ long and $20 \mathrm{~mm}$ diameter was successfully grown by Sankaranarayanan-Ramasamy (SR) method. The lattice parameter values were determined from the single crystal XRD and it is shown that it belongs to orthorhombic crystal system with the space group $\mathrm{P} 22_{1} 2_{1} 2_{1}$. Transmission spectral analysis shows that the transmittance percentage of SR grown crystal was $23 \%$ higher than that of conventionally grown crystal. The observed high intensity in the blue region for SR method grown crystal, indicates its high crystalline perfection and outcome compared to conventionally grown crystal. The laser damage threshold studies indicate that the SR method grown crystal has has good optical tolerance due to its crystalline perfection. Though both the crystal exhibits negative photoconductive nature, the photosensitivity is greater for SR method grown crystal. Higher thermal diffusivity in SR grown crystal is a favourable property for NLO materials, since the heat dissipation is faster during high power laser irradiation.

\section{Acknowledgements}

The authors are thankful to SAIF, IIT-Madras for single crystal XRD analysis. We thank NCIF, National College, Tiruchirappalli for hardness studies and also we thank to ACIC, St. Joseph's College, Tiruchirappalli for PL measurement. We thank Dr. S. Kalainathan, Centre for Crystal Growth, VIT University, Vellore for LDT results.

\section{References}

1. D. Wang, G. Zhou, X. Xu, X. Wang, H. Liu, Y. Ren, Z. Shao, M. Jiang, Opt. Laser Technol.34 (2002) 343-346.

2. J. Badan, R. Hierle, A. Perigaud, J. Zuss (Eds.), American Chemical Symposium Series 233, American Chemical Society, Washington. DC, 1993.

3. H. Q. Sun, D. R. Yuan, X. Q. Wang, X. F. Cheng, C. R. Gong, M. Zhou, H. Y. Xu, X. C. Wei, C. N. Luan, D. Y. Pan, Z. F. Li, X. Z. Shi, Cryst Res Technol. 40 (2005) 882-886.

4. F. Barati, H. RezagholipourDizaji, Opt. Quant. Electron (2016) 48:432.

5. R. Bairava Ganesh, V. Kannan, R. Sathyalakshmi, P. Ramasamy, Mater. Lett.61 (2007) 706-708.

6. K.Sankaranarayanan, P.Ramasamy, J. Crystal Growth 280 (2005) 467-473.

7. K. Selvaraju, K. Kirubavathi, N. Vijayan, S. Kumararaman, Mod. Phys. Lett. B 23 (2009) 861-869. 
8. R. Sathyalakshmi1, V. Kannan1, R. Bairava Ganesh1, and P. Ramasamy, Cryst. Res. Technol. 42, No. 1, 78 83 (2007) / DOI 10.1002/crat.200610775

9. J. Uma, V.Rajendran, Progress in Natural Science:Materials International 26 (2016)24-31

10. M. Delfino, J.P. Dougherty, W.K. Zwicker, M.M. Choyk, J. Cryst. Growth 36 (1976) 267-272

11. Wang Yan, Yu Xi-ling and Yin Shao Tang, Cryst.Res. Technol, Vol 40, (2005), 768-772.

12. A. L. Fahrenbruch and R.H. Bude, Fundamentals of Solar Cells, Academic, New York, 1983.

13. J.Tauc, R.Grigorovici, A.Vancu, Optical properties and electronic structure of Amorphous Germanium, Phys.Status Solidi. (b) 15 (1966) 627-637

14. P. Karuppasamy, V. Sivasubramani,M. Senthil Pandian and P. Ramasamy, RSC Adv., 6 (2016) 109105109123.

15. M.Parthasarthy, R.Gopalkrishnan, J. Cryst. Growth 372 (2013) 100-104

16. N. Vijayan, G. Bhagavannarayana, K. R. Ramesh, R. Gopalakrisnan, K. K. Maurya and P. Ramasamy, Cryst. Growth Des. 6 (2006) 1542-1546.

17. H.L.Bhat, Growth and characterization of some novel crystals for nonlinear optical applications, Bull. Mater. Sci., 17 (1994) 1233-1249
18. I. M. Ashraf, H. A. Elshaikh and A. M. Badr, Photoconductivity in $\mathrm{Tl}_{4} \mathrm{~S}_{3}$ layered single crystals, Cryst. Res. Technol.39 (2004) 63-70.

19. V. N. Joshi, Photoconductivity; Macrel Dekker.New York, 1990

20. T. Somasundaram, P. Ganguly, and C. N. R. Rao, Journal of Physics - C, 19, (1986), 2137.

21. S.A.Martin Britto Dhas, E.Ramachandran, P. Raji, K. Ramachandran, and S.Natarajan, Crystal Research Technology, 42(6), (2007), 601 - 606.

22. P. Raji, C. Sanjeeviraja, and K. Ramachandran, Crystal Research Technology 39, (2004), 617.

23. W.L. Barros Melo and R. M. Faria, Applied Physics Letter, 67, (1995), 3892-3894.

24. J. D. Jackson, Classical Electrodynamics, Wiley Eastern. 1978, 321.

25. D. R. Penn, Wave-number-dependent dielectric function of semiconductors, Phys. Rev., 128 (1962) 2093-2097.

26. C. Balarew, R. Duhlew, Synthesis, Optical and Dielectric Properties of Tris-Glycine Zinc Chloride (TGZC) Single Crystals, J. Solid-State Chem. 55 (1984) 1-6.

27. A. Dev, S. Chakrabarti, S. Kar and S. Chaudhuri, Journal of Nanoparticle Research, 7, (2005), 195-201

\section{How to cite this article:}

Jayanthi L and Prabavathi N (2017) ' Directional Growth Of L-Glutamic Acid Hydrochloride (Lghcl) Single Crystals Grown By Conventional And Sankaranarayanan-Ramasamy (Sr) Method: A Comparative Study By Means Of Optical, Ldt, Photoconductivity, Photo Acoustic And Polarizability Properties', International Journal of Current Advanced Research, 06(05), pp. 3927-3932. DOI: http://dx.doi.org/10.24327/ijcar.2017.3932.0399 